\title{
Jobannes Paulmann
}

\section{Deutschland in der Welt: Auswärtige Repräsentationen und reflexive Selbstwahrnehmung nach dem Zweiten Weltkrieg- eine Skizze}

Montreal 1967: Als das Ausstellungsgelände für die Weltausstellung in Montreal, das auf zwei künstlich vergrößerten Inseln im St.-Lawrence-Strom lag, sich noch im Aufbau befand, fuhr ein deutsches Handelsschiff vorbei. Sein Dritter Offizier erblickte etwas, das ihn dazu veranlaßte, ein Foto zu schießen und nach seiner Heimkehr an den „Sehr geehrten Herrn Außenminister" zu schicken. Bei dem Objekt, das ihm ins Auge gesprungen war, handelte es sich, wie der Herr Außenminister sicher bemerke, um eine "deutsche Flagge“, die über dem Gelände der Weltausstellung wehte. „Da es die einzige aufgezogene Nationalflagge war, fiel sie sofort auf", betonte der besorgte Offizier; Schiffe aller Nationen verkehrten dort, und er glaube kaum, „daß wir als Deutsche [damit] einen guten Eindruck hinterlassen" 1 .

Flagge-Zeigen als unangemessene Handlung, man sollte annehmen, daß dies im Bonner Außenministerium nicht weiter beachtet worden wäre: Tatsächlich geschah das Gegenteil. Der VLR I Dr. v. Bismarck-Osten in der handelspolitischen Abteilung des Auswärtigen Amts stellte verschiedene Theorien auf ${ }^{2}$. Sollte es sich lediglich um eine optische Täuschung handeln? Zwei verschiedene Wimpel könnten perspektivisch verkürzt übereinander geweht haben, so daß der Eindruck entstand, es handele sich um eine deutsche Flagge. Oder hatte jemand das Schwarz hineinretuschiert? Eine Provokation, aus dem Osten gar? Das Generalkonsulat in Montreal konnte auf Anfrage den ,Fall rasch aufklären. Zum fraglichen Zeitpunkt war für den deutschen Pavillon Richtfest gefeiert worden, und zu diesem

1 PA/AA [Politisches Archiv des Auswärtigen Amts, Berlin] IIIA1, Bd. 207: G. R. an Außenminister, 24. Oktober 1966 (hdschrftl.) - Die Anmerkungen zu diesem Beitrag, der ein Forschungsprogramm umreißt, sind nicht auf Vollständigkeit angelegt, sondern beschränken sich auf Belege und weiterführende Hinweise. Heide Fehrenbach, Madeleine Herren und Dirk Schumann danke ich für kritische Anregungen.

2 Ebd., Marginalie Bismarck-Osten, 27. Oktober 1966, und Generalkonsulat an AA, Montreal, 17. November 1966 (Betr. Hissung der deutschen Flagge auf dem Gelände der Weltausstellung von 1967). 
Anlaß hatten auch andere Nationen bereits zuvor ihre Flaggen gesetzt. Inzwischen wehten im übrigen über fast allen Gebäuden die Nationalflaggen der jeweiligen Aussteller.

All dies erläuterte Bismarck-Osten dem Offizier der Handelsmarine in seinem Antwortschreiben. Er fügte einige Bemerkungen an, die den Vorfall für eine Geschichte des deutschen Selbstverständnisses nach dem Zweiten Weltkrieg signifikant erscheinen lassen: „Sie haben [...] durchaus ernst zunehmende Bedenken dagegen geäussert, daß die deutsche Flagge über dem Gelände [...] in Montreal gezeigt wird. [...] wir Deutsche [müssen] gerade im Ausland alles unterlassen, was zu einer Kritik uns gegenüber Anlass geben könnte." Der vorgebrachte Fall gebe aber keinen Anlaß zur Kritik, denn es sei allgemein üblich, nach Fertigstellung des Rohbaus die Landesflagge zu setzen. Auch die anderen hätten dies getan. „Die Bundesrepublik", so der VIR I, "hat sich also insoweit nur einem allgemeinen Brauch angepasst." 3

Die Aufregung des Offiziers der Handelsmarine und die Erklärungen des adeligen Beamten im Auswärtigen Amt beruhten auf einer beiden gemeinsamen Verhaltensnorm: dem Bestreben, als Deutsche im Ausland zurückhaltend aufzutreten, auch und vielleicht gerade dann, wenn es um nationale Selbstdarstellung ging; diese Norm betrachteten beide Männer als wesentliche Grundlage für ihr praktisches Handeln. Eine solche Haltung war allerdings für Deutsche, selbst nach 1945, nicht immer selbstverständlich gewesen. Eine Episode, die sich nur ein knappes Jahrzehnt früher zu einem ähnlichen Anlaß abspielte, soll dies illustrieren.

Der Publizist und Heidelberger Politologe Dolf Sternberger reiste 1958 zur ersten Weltausstellung, die nach dem Zweiten Weltkrieg stattfand, nach Brüssel. In einem Feuilleton, das er in der Halbmonatsschrift „Die Gegenwart“ über seinen Aufenthalt publizierte, schilderte er, wie er eines Tages im Hotel einen Deutschen beobachtete, „sportlich gekleidet, mit offenem Kragen“, der mit seinen beiden Begleitern kein Zimmer mehr erhielt, weil die zentrale, amtliche Quartiervermittlung „Logexpo“ offenbar einen Fehler gemacht hatte. Dem freundlichen belgischen Portier gelang es nicht, den enttäuschten deutschen Gast zu beruhigen. Der wollte sich mit der angebotenen Erklärung und Entschuldigung nicht zufrieden geben: „Wenn das eine so unfähige Organisation ist, soll man sie nicht einsetzen. [...] Unser Geld können sie nehmen, aber jetzt stehen wir da zu Dritt und haben keine Unterkunft. Eine schöne Dienststelle, das!"4 Sternberger kommentierte das Auftreten seines Landsmannes in der "Gegenwart“ und verurteilte es zugleich, indem er über die Sprache eine Verbindung zur gerade 13 Jahre zurückliegenden NS-Vergangenheit herstellte: „Da waren sie also, die legendären ,Deutschen im Ausland'. So leibhaftig hatte ich's noch nicht erfahren. Organisation, Einsatz, Dienststelle. Es fehlt gerade nur die Erinnerung, daß es unter der deutschen Besat-

${ }^{3}$ Ebd., Bismarck-Osten an G. R., 24. November 1966.

4 d. st. (Dolf Sternberger), Meine "Deutschen Tage" in Brüssel, in: Die Gegenwart Nr. 315, 13. Jg., v. 28. Juni 1958, 401-403, hier 401. 
zung gewiß besser geklappt hätte. [...] Bedrückt suchte ich mein Zimmer auf. "5 Der Publizist bezog mit seiner Schilderung deutlich Stellung gegen die Stimmung des, Wir sind wieder Wer!' und bedauerte indirekt das Fehlen eines Moments, das neben Verwestlichung und wachsendem Wohlstand das Selbstverständnis der Westdeutschen langfristig prägen sollte. Sternberger benannte nicht ausdrücklich, was er vermißte. Seine Kommentare sowie sein eigenes, im Bericht geschildertes Auftreten ließen aber die erwünschte Grundhaltung, die in der Episode von 1958 nur durch Absenz auffiel, erkennen: Diese noch unerfüllte Norm, ein charakteristisches, wenn auch nicht unumstrittenes Moment westdeutschen Verhaltens nach dem Zweiten Weltkrieg, möchte ich als die ,Haltung der Zurückhaltung' bezeichnen'.

Sternbergers Feuilletonbeitrag, der auch als Mahnung an seine Leserinnen und Leser zu verstehen war, läßt vermuten, daß diese besondere Verhaltensnorm 1958 noch nicht eingeübt war, während der Vorfall von 1967 auf eine insgesamt breitere Akzeptanz der Haltung der Zurückhaltung hinweist. Diese Hypothese läßt sich einerseits durch den Verweis auf die anerkannt zurückhaltende Außenpolitik der Bundesrepublik Deutschland bis zur Vereinigung von 1990 stützen. Die publizistischen Beiträge vor allem zu Anfang der 90er Jahre, die ein neues Verhältnis der Deutschen zur Macht in der internationalen Politik einklagten ${ }^{7}$, scheinen sich als eine Minderheitenposition erwiesen zu haben, deren Vertreter glaubten, gegen die vorherrschende Grundausrichtung anschreiben zu müssen. Andererseits wird die Hypothese einer nach dem Ende des Zweiten Weltkriegs zunächst umstrittenen Norm der Zurückhaltung, die sich erst allmählich durchsetzte, durch andere Episoden aus der Geschichte der frühen Bundesrepublik gestützt. Erinnert sei an die

\section{Ebd. 401.}

${ }^{6}$ Dieser Stil der Selbstdarstellung zog sich gleichsam als Leitmotiv durch die offiziellen (west)deutschen Beteiligungen in Brüssel und Montreal. Ich nehme hier eine Selbstbeschreibung aus der Monatszeitung des Deutschen Werkbundes auf, dessen Vertreter maßgeblich an der Konzeption des deutschen Beitrags zur Weltausstellung in Brüssel beteiligt gewesen waren - siehe Ernst Johann, Die Haltung der Zurückhaltung. Deutschland in Brüssel, in: werk und zeit 7/6 (1958) 3-6. Zum deutschen Beitrag für Brüssel siche jetzt Christopher Oesterreich, Umstrittene Selbstdarstellung. Der deutsche Beitrag zur Weltausstellung in Brüssel 1958, in: VfZ 48 (2000) 127-153, und Paul Sigel, Exponiert. Deutsche Pavillons auf Weltausstellungen (Berlin 2000) 173-206.

7 Siehe etwa Hans-Peter Schwarz, Die gezähmten Deutschen. Von der Machtbesessenheit zur Machtvergessenheit (Stuttgart 1985), sowie ders., Die Zentralmacht Europas. Deutschlands Rückkehr auf die Weltbühne (Berlin 1994), und vor allem Gregor Schöllgen, Angst vor der Macht. Die Deutschen und ihre Außenpolitik (Berlin 1993); vgl. dagegen Hans-Ulrich Wehler, Angst vor der Macht? Die Machtlust der neuen Rechten (Friedrich-Ebert-Stiftung, Gesprächskreis Geschichte 8, Bonn 1995) wiederabgedr. in: Christian Jansen, Lutz Nietbammer, Bernd Weisbrod (Hrsg.), Von der Aufgabe der Freiheit. Politische Verantwortung und bürgerliche Gesellschaft im 19. und 20. Jahrhundert (Fs. Hans Mommsen, Berlin 1995) 309319. Auch neuere geschichtswissenschaftliche Darstellungen der deutschen Außenpolitik seit der Reichsgründung legen nach dem Zweiten Weltkrieg Zurückhaltung bzw. Mäßigung als Beurteilungskriterium zugrunde - besonders deutlich bei Klaus Hildebrand, Das vergangene Reich. Deutsche Außenpolitik von Bismarck bis Hitler 1871-1945 (Stuttgart 1995) 149-155, 895 f. u.ö. 
konträren politischen Stile Konrad Adenauers und Kurt Schumachers in Fragen nationaler Interessenvertretung gegenüber den alliierten Besatzungsmächten oder an die tadelnden heimischen Reaktionen auf das Singen der ersten Strophe der Nationalhymne, nachdem die deutsche Mannschaft in Bern 1954 die Fußballweltmeisterschaft gewonnen hatte.

Professionelle auswärtige Berichterstatter bemerken gelegentlich, daß die Beschäftigung mit dem eigenen Bild im Ausland geradezu eine Obsession der Deutschen sei ${ }^{8}$. Jenseits von Hypothesen, die sich auf gegenwärtige Beobachtungen und historische Episoden stützen, wissen wir allerdings wenig darüber, wie das Verhältnis zum Ausland die Entwicklung des deutschen Selbstverständnisses nach dem Zweiten Weltkrieg langfristig prägte ${ }^{9}$. Im folgenden wird ein Forschungsprogramm skizziert, das die Mechanismen und Wirkungen der Beschäftigung mit dem deutschen Selbstbild im Ausland historisch zu analysieren und erklären verspricht. In Umrissen soll hier der Zusammenhang erläutert werden, in dem die beiden Episoden von 1958 und 1967 ihre Bedeutung gewannen. Sie werden dabei auf zweifache Weise weniger anekdotisch, als sie den Leserinnen und Lesern zunächst erschienen sein mögen: Einerseits zeigen die erwähnten Fälle bestimmte Meinungen und Verhaltensweisen deutscher Beobachter und Repräsentanten an, fungieren damit für uns als Indikatoren historischer Entwicklungen und Gefühlslagen. Anderseits erlangen sie Bedeutung als historische Momente, in denen und durch die bestimmte Einstellungen und Praktiken eingeübt und verfestigt wurden; sie werden damit als Faktoren eines mentalitätsgeschichtlichen Prozesses greifbar.

Worum gebt es? Im Mittelpunkt des Interesses steht die Selbstdarstellung der Bundesrepublik Deutschland im Ausland. Die Aufmerksamkeit richtet sich dabei auf die Debatten, die innerhalb der Bundesrepublik darüber geführt wurden, wie sich der westliche Teilstaat außerhalb der eigenen Grenzen präsentieren solle. Was würde ,das Ausland über die deutsche Art des Auftretens denken? Welche Reaktionen waren im Inland zu erwarten? Diese Fragen stellten sich die Zeitgenossen nach dem Zweiten Weltkrieg in der ein oder anderen Form immer wieder.

8 Deidre Berger, Learning to stop hating Germans: the challenge of journalistic objectivity, in: Scott Denham, Irene Kacandes, Jonathan Petropoulos (Hrsg.), A user's guide to German cultural studies (Ann Arbor, MI 1997) 93-111, hier 99.

9 Siche aber die neueren Forschungen zu Amerikanisierung, Westernisierung und Sowjetisierung, die insbesondere für Westdeutschland verschiedene Felder jenseits der diplomatischen und wirtschaftlichen Integration behandeln: Anselm Doering-Mantenffel, Wie westlich sind die Deutschen? Amerikanisierung und Westernisierung im 20. Jahrhundert (Göttingen 1999); Heide Febrenbach, Uta G. Poiger (Hrsg.), Transactions, transgression, transformations. American culture in Western Europe and Japan (New York 2000); Konrad Jarausch, Hannes Siegrist (Hrsg.) Amerikanisierung und Sowjetisierung in Deutschland 1945-1970 (Frankfurt a.M. 1997) und Michael Lemke (Hrsg.), Sowjetisierung und Eigenständigkeit in der SBZ/DDR (1945-1953) (Köln 1999). Vgl. auch die neueren Thesen zum postkolonialen Erbe in Deutschland in Sara Friedrichsmeyer, Sara Lennox, Susanne Zantop (Hrsg.), The imperialist imagination. German colonialism and its legacy (Ann Arbor, MI 1998) 1-7, $24 \mathrm{f}$. und die Aufsätze in Teil 3 dieses Sammelbandes. 
Die Antworten fielen unterschiedlich aus, und die Diskussion darüber veränderte das gesellschaftliche und politische Selbstverständnis ${ }^{10}$. Verhaltensnormen und -muster in Westdeutschland wurden auf diese Weise langfristig durch den Bezug auf das Ausland geprägt.

Das Nachdenken über das im Ausland präsentierte Selbstbild und dessen mögliche Fremdbeurteilung läßt sich als ,Wechselspiel der Eigenwahrnehmung' beschreiben ${ }^{11}$, das wesentlich dazu beitrug, daß sich in der Zeit vom Kalten Krieg bis zur Entspannungspolitik allmählich eine neue Identität und Mentalität, ein spezifisch bundesrepublikanisches Selbstbewußtsein ausbildete. Die politischen, sozialen und kulturellen Grundlagen dieses Vorgangs bleiben historisch zu untersuchen $^{12}$. Mehrere Faktoren bestimmten dabei den Rahmen für die auswärtigen Repräsentationen. Allgemein ist zu klären, inwieweit die verschiedenen Aspekte des Selbstverständnisses, zu denen etwa die eingangs erwähnte Haltung der Zurückhaltung gehörte, von außen erzwungen waren, auf Einsicht in Notwendigkeiten beruhten oder vorhandenen Überzeugungen entsprangen. Nach dem Zweiten Weltkrieg galt es gerade gegenüber dem Ausland, sich von der Politik der nationalsozialistischen Herrschaft sichtbar abzusetzen, insbesondere auch weil Deutschland in den vergangenen Jahren versucht hatte, die Welt zu beherrschen. Aufgrund der Teilung spielte ferner die Systemkonkurrenz mit der DDR eine zentrale Rolle dafür, wie die Bundesrepublik sich und, vor allem in den ersten Jahrzehnten, vom Anspruch her Deutschland als Ganzes nach außen repräsentierte. Schließlich prägten auch die Vorstellungen von einer fortschreitenden Modernisierung, welche die Gesellschaft veränderte, das Selbstbild. Abkehr von einem Teil der deutschen Geschichte, Abwehr des anderen Teilstaates und Modernität als Zielvorstellung bildeten das Spannungsfeld der allgemeinen historischen

10 Die Selbstdarstellung der DDR im Ausland stellt gleichfalls einen lohnenden Gegenstand dar, doch sind die Rückwirkungen auf das gesellschaftliche und politische Selbstverständnis aufgrund der eingeschränkten Öffentlichkeit schwer zu erfassen. Dic ostdeutschen Repräsentationen finden daher zunächst nur insofern Berücksichtigung, als sie im Westen als Konkurrenz oder Folie wahrgenommen wurden. Die methodischen Probleme werden deutlich bei Ina Rossow, ,... . alles nett, schön und gefühlsbetont, mit viel Absicht.“ Die III. Weltfestspiele der Jugend und Studenten 1951 im Kalten Krieg, in: Fortschritt, Norm und Eigensinn. Erkundungen im Alltag der DDR, hrsg. v. Dokumentationszentrum Alltagskultur der DDR e.V. (Berlin 1999) 17-37; dies., „Rote Ohren, roter Mohn, sommerheiße Diskussion.“ Die X. Weltfestspiele der Jugend und Studenten 1973 als Möglichkeit für vielfältige Begegnungen, in: ebd. 256-275; und Gerhard Naumann, Die III. Weltfestspiele der Jugend und Studenten 1951 in Berlin. Vorgeschichte - Verlauf - Ergebnisse, in: ZfG 35 (1987) 209-217; zu den Selbstinszenierungen im eigenen Land siehe ferner Monika Gibas, Rainer Gries, Barbara Jakoby, Doris Müller (Hrsg.), Wiedergeburten. Zur Geschichte der runden Jahrestage der DDR (Leipzig 1999).

11 Vgl. hierzu die „Wechselspiele der Fremdwahrnehmung“ bei Jürgen Osterhammel, Die Entzauberung Asiens. Europa und die asiatischen Reiche im 18. Jahrhundert (München 1998) 81-84.

12 Vgl. die Thesen, die Edgar Wolfrum vornehmlich am Beispiel des Gedenktages zum 17. Juni 1953 entwickelt - Edgar Wolfrum, Geschichtspolitik in der Bundesrepublik Deutschland. Der Weg zur bundesrepublikanischen Erinnerung (Darmstadt 1999). 
Bedingungen, unter denen die Debatte über die auswärtige Selbstdarstellung der Bundesrepublik Deutschland stattfand.

An welchen konkreten Gegenständen läßt sich die reflexive Selbstwabrnebmung untersuchen? Das breite Feld von der Wirtschaft über die Gesellschaft bis zur Kultur, welches nicht der klassischen Diplomatie angehört, hat im Rahmen einer Entwicklungsgeschichte der internationalen Beziehungen und mit Blick auf die Vorgeschichte der Globalisierung in jüngster Zeit vermehrt wissenschaftliche Aufmerksamkeit gefunden ${ }^{13}$. Die auswärtige Repräsentation und die damit verbundene reflexive Selbstwahrnehmung beschränkten sich gleichfalls nicht auf diplomatische Vertretungen, sondern fanden in vielfältigen Formen und in verschiedenen Bereichen statt, die von der historischen Forschung bislang allerdings fast gar nicht beachtet wurden. Im Vordergrund stehen daher zunächst die Teilnahme an internationalen Industriemessen, die Beteiligung an Weltausstellungen und die auswärtige Kulturpolitik; neben diesen ökonomischen und kulturellen Formen sollen mit den Auslandsreisen hochrangiger Vertreter der Bundesrepublik ferner im engeren Sinne politische Selbstdarstellungen analysiert werden ${ }^{14}$. Die Olympischen Spiele von 1972 stellen schließlich ein besonderes Ereignis dar, bei dem sich die Bundesrepublik auf eigenem Boden der Welt präsentierte. Das Wechselspiel der Eigenwahrnehmung im Spannungsfeld von Abkehr, Abwehr und Modernität manifestierte sich bei dieser Gelegenheit knapp drei Jahrzehnte nach Kriegsende wie unter einem Vergrößerungsglas ${ }^{15}$.

Die genannten Felder auswärtiger Repräsentation bilden exemplarische Gegenstände; sie waren lediglich herausragende Beispiele für die Art, wie Westdeutschland sich in der Welt darstellte. Die möglichen Untersuchungsgegenstände lassen sich leicht vermehren, wobei ein breites Spektrum berücksichtigt werden kann: von staatlicher Repräsentation (etwa im Bau von Botschaftsgebäuden ${ }^{16}$ ) und der Vertretung gesellschaftlicher Gruppen (wie etwa der Kirchen im Ökumenischen

13 Siehe Adam Watson, The cvolution of international society (London 1992); Michael Geyer, Charles Bright, World history in a global age, in: AHR 100 (1995) 1034-1060; Akira Iriye, Cultural internationalism and world order (Baltimore, MD 1997) und Martin $\mathrm{H}$. Geyer, Johannes Paulmann (Hrsg.), The mechanics of internationalism. Culture, society, and politics from the 1840s to the First World War (Oxford 2001).

14 Vgl. zum 19. Jahrhundert Johannes Paulmann, Pomp und Politik. Monarchenbegegnungen in Europa zwischen Ancien Régime und Erstem Weltkrieg (Paderborn 2000).

is So hieß es in einem Erlaß Außenminister Scheels an die Auslandsvertretungen vom 16. April 1970: „Die Olympischen Sommerspiele 1972 in München und Kiel werden noch mehr als bisher die Aufmerksamkeit der Welt auf die Bundesrepublik Deutschland lenken. [...] Die Erinnerung an [...] unsere historische Vergangenheit und nicht zuletzt das Bewußtsein unserer besonderen politischen Lage werden dabei cine nicht unerhebliche Rolle spielen. [...] Somit bietet sich für uns eine einmalige Gelegenheit, [...] dem Ausland ein Bild des modernen Deutschlands [sic!] mit allen seinen politischen, wirtschaftlichen und kulturellen Aspekten zu vermitteln." - abgedr. in: Auswärtiges Amt (Hrsg.), Die Auswärtige Politik der Bundesrepublik Deutschland (Köln 1972) Nr. 291.

16 Botschaften. 50 Jahre Auslandsbauten der Bundesrepublik Deutschland. Fine Ausstellung des Deutschen Architekturmuseums Frankfurt am Main, hrsg. v. Olaf Asendorf (Tübingen 2000). 
Rat oder diejenige von Wissenschaftsverbänden in internationalen Organisationen), über die kommerzielle Werbung (etwa für Reisen nach Deutschland, in deutsche Regionen und Städte) und die künstlerische Repräsentation im Ausland (etwa auf Filmfestivals ${ }^{17}$ ) bis hin zum - mit Quellen wohl nur episodisch erfaßbaren - Auftreten einzelner Deutscher (u.a. als Touristen).

Das Interesse an der reflexiven Selbstwahrnehmung erfordert nicht, die genannten Aktivitäten Deutscher im Ausland per se zu untersuchen. Vielmehr genügt es, zentrale Ereignisse, grundlegende Debatten oder Konfliktfälle auszuwählen, an denen sich eine Diskussion entzündete. Es geht also etwa im Zusammenhang der auswärtigen Kulturpolitik nicht darum, eine Darstellung der Mittlerorganisationen wie des Goethe-Instituts, des DAAD oder von Inter Nationes zu schreiben ${ }^{18}$. Mit Blick auf das Selbstverständnis in der Bundesrepublik sind statt dessen etwa die Erklärungen anläßlich der Bildung eines Kulturbeirates für das Auswärtige Amt im Jahre $1961^{19}$, die Reaktionen auf kulturpolitische Aktivitäten der DDR in der Dritten Welt seit der zweiten Hälfte der fünfziger Jahre ${ }^{20}$, die parlamentarischen Beratungen von 1970 über das Einsetzen einer Enquête-Kommission „Auswärtige Kulturpolitik" 21 oder aber Kontroversen zu analysieren wie

17 Zur Bedeutung des Berliner Film-Festivals in diesem Zusammenhang siehe Heide Fehrenbach, Cinema in democratizing Germany. Reconstructing national identity after Hitler (Chapel Hill, NC 1995) 234-253. Der Termin der ersten Berlinale wurde 1951 gezielt in den Juni gelegt, um damit das Film-Festival, das einem der Initiatoren zufolge die "demokratischste Kunstform" darbot, als Gegengewicht zu den Weltfestspielen der Jugend und Studenten im Ostsektor der Stadt wirken zu lassen - ebd. $238 \mathrm{f}$.

18 Zur Geschichte des DAAD liegt jetzt ein wissenschaftliches Sammelwerk vor: Deutscher Akademischer Austauschdienst (Hrsg.), Spuren in die Zukunft. Der deutsche Akademische Austauschdienst 1925-2000, 3 Bde. (Bonn 2000), bes. Bd. 1. Der DAAD in der Zeit. Geschichte, Gegenwart und zukünftige Aufgaben, hrsg. v. Peter Alter; in Kürze erscheint die mit dem Rave-Forschungspreis 2003 ausgezeichnete Dissertation von Ulrike Stoll, Kulturpolitik als Beruf. Dieter Sattler in München, Rom und Bonn (1906-1968). Sattler war 19521959 Kulturattaché am Quirinal in Rom und leitete von 1959 bis 1966 die Kulturabteilung des Auswärtigen Amtes.

${ }^{19}$ Hans-Peter Schwarz (Hrsg.), Handbuch der deutschen Außenpolitik (München 1975) 756; und Dieter Braun (Hrsg.), Deutsche Kulturpolitik im Ausland: 1955 bis heute. Dokumente, Kommentare, Tendenzen (Schriftenreihe des Goethe-Instituts 2, München 1966).

${ }^{20}$ Siehe zur kulturpolitischen Konkurrenz Heinrich End, Zweimal deutsche Außenpolitik. Internationale Dimensionen des innerdcutschen Konflikts 1949-1972 (Köln 1973) 130-132; Anita M. Mallinckrodt, Die Selbstdarstellung der beiden deutschen Staaten im Ausland. ,Image-Bildung' als Instrument der Außenpolitik (Köln 1980), und Hans-Joachim Spranger, Lothar Brock, Die beiden deutschen Staaten in der Dritten Welt. Die Entwicklungspolitik der DDR - eine Herausforderung für die Bundesrepublik Deutschland (Opladen 1987) 270275; zur Kulturpolitik der DDR allgemein Hans Lindemann, Kurt Müller, Auswärtige Kulturpolitik der DDR. Die kulturelle Abgrenzung der DDR von der Bundesrepublik Deutschland (Bonn 1974); Peter Lübbe, Kulturelle Auslandsbeziehungen der DDR. Das Beispiel Finnland (Bonn 1981) und H. Peisert, J. Kuppe, Kulturpolitik, auswärtige, in: Wolfgang $R$. Langenbucher, Ralf Rytlewski, Bernd Weyergraf (Hrsg.), Kulturpolitisches Wörterbuch Bundesrepublik Deutschland / Deutsche Demokratische Republik im Vergleich (Stuttgart 1983) 372-379.

21 Bartbold C. Witte, Die Enquête-Kommission des Bundestages - Anmerkungen zu ihrem 
diejenige, die 1972 dazu führte ${ }^{22}$, daß Heinrich Böll seine Zusammenarbeit mit dem Goethe-Institut öffentlich aufkündigte.

Die Art des Vorgehens und die Auswahl der Gegenstände sind ferner dadurch gekennzeichnet, daß nach Äußerungen des Selbstverständnisses dort gesucht wird, wo dies für die Zeitgenossen nicht explizit Thema sein mußte. Die Intellektuellendebatten über deutsche Identität oder die Vorstellungen einer wissenschaftlichen Disziplin wie der Soziologie über die deutsche Gesellschaft interessieren also nur am Rande ${ }^{23}$. Auch im Kontrast zur Analyse solcher Reflexionen werden über die hier ausgewählten Gegenstände die Medien und Strategien der Selbstdarstellung stärker als bisher beachtet, denn symbolisches Handeln, Repräsentation durch Gegenstände, Architektur und künstlerische Produktionen gehörten ebenso zu den möglichen Ausdrucksformen wie Parlamentsreden oder Zeitschriftenartikel.

Das Beschicken internationaler Industriemessen durch westdeutsche Firmen etwa enthielt indirekt Elemente deutscher Selbstdarstellung. Beispielsweise konnte die noch von der amerikanischen Militärregierung unterstützte „German Industrial Fair", die im Frühjahr 1949 in New York stattfand, nicht nur eine, sachliche' Darbietung deutscher Industrieprodukte sein und der Rekonstruktion der ökonomischen Außenbeziehungen dienen, sondern berührte, wie die Diskussion amerikanischer Juden über die Forderung nach einem Boykott zeigte, die Wahrnehmung Deutschlands im weiteren Sinne ${ }^{24}$. Sachlichkeit' selbst stellte ein bestimmtes, keineswegs wertfreies Selbstverständnis dar ${ }^{25}$. Erinnert sei in diesem Zusammenhang auch an die indirekten Inszenierungen des jeweiligen Systemerfolgs in den Messen von Hannover und Leipzig ${ }^{26}$. Teilweise wirkten politische Vorgänge aber auch unmittelbar auf die Veranstaltung von Industriemessen ein,

Bericht über auswärtige Kulturpolitik, in: Kurt Düwell, Werner Link (Hrsg.), Deutsche Auswärtige Kulturpolitik seit 1871. Geschichte und Struktur (Köln 1981) 295-342.

22 Wolfgang Jäger, Werner Link, Republik im Wandel 1974-1982. Die Ära Schmidt (Geschichte der Bundesrepublik 5/II, Stuttgart 1987) 416.

${ }^{23} \mathrm{Vgl}$. Paul Nolte, Die Ordnung der deutschen Gesellschaft. Selbstentwurf und Selbstbeschreibung im 20. Jahrhundert (München 2000) $23 \mathrm{f}$. u. Kap. IV, und exemplarisch für dic Debatten der 1980er Jahre Werner Weidenfeld (Hrsg.), Die Identität der Deutschen (München 1983).

24 Peter Nowick, The Holocaust in American Life (Boston, MA 1999) 96f.; allgemeiner siehe Hanno Sowade, Wegbereiter des Aufstiegs. Die Industrie und Handelskammern und die Rekonstruktion der Außenbeziehungen der westdeutschen Wirtschaft 1945-1949/50 (München 1992), sowie Jonathan Wiesen, Overcoming Nazism. Big business, public relations, and the politics of memory, 1945-1950, in: CEH 29 (1996) 201-226.

25 Vgl. Willibald Steinmetz, Anbetung und Dämonisierung des, Sachzwangs‘. Zur Archäologie einer deutschen Redefigur, in: Micbael Jeismann (Hrsg.), Obsessionen. Beherrschende Gedanken im wissenschaftlichen Zeitalter (Frankfurt a.M. 1995) 293-333.

26 Siehe die Selbstdarstellung von Dieter Tasch, 50 Jahre Zukunft. Messen in Hannover 1947-1997, hrsg. v. Deutsche Messe AG (Hannover 1997) sowie die ersten Ergebnisse eines wissenschaftlichen Projektes zur Leipziger Messe in Hartmut Zwabr, Thomas Topfstedt, Günter Bentele (Hrsg.), Leipzigs Messen 1497-1997, 2 (Köln 1997); ferner aus unternchmensgeschichtlicher Perspektive auch Marcus Schüller, Wiederaufbau und Aufstieg der Kölner Messe 1946-1956 (Stuttgart 1999). 
wie das Beispiel der für 1961 in Moskau geplanten Deutschen Industrieausstellung belegt ${ }^{27}$. Die Interessen der Unternehmen deckten sich dabei nicht notwendigerweise mit denjenigen der offiziellen Außenpolitik, was unter anderem an den negativen Folgen der Hallstein-Doktrin für die Partizipation deutscher Firmen an Ausstellungen in Entwicklungsländern zutage trat ${ }^{28}$.

Neben der Konzentration auf auswärtige Repräsentationen, die im Innern Debatten hervorriefen, und neben dem Interesse an den eingesetzten Medien und Strategien wird bei der Auswahl der Gegenstände ein drittes Kriterium angewendet: Gelegenheiten, bei denen die Selbstdarstellung gegenüber dem Ausland, aus sachlichen Gründen, nicht leicht fiel, lassen die Probleme deutschen Selbstverständnisses besonders scharf hervortreten. Auf dem Felde der Auslandsreisen hochrangiger Vertreter der Bundesrepublik ist dabei etwa an die Begegnung Adenauers mit Ben Gurion, an seine Zusammenkunft mit de Gaulle in Reims oder aber, als Beispiel einer außergewöhnlichen symbolischen Geste, an den Kniefall Brandts in Warschau zu denken. Auf diesem Wege findet auch die unmittelbar vorangehende Periode deutscher Geschichte Eingang in die Erörterungen. Die zeitgenössische Auseinandersetzung mit deutschen Traditionen und insbesondere mit der nationalsozialistischen Vergangenheit prägten das Selbstverständnis ausgesprochen und unausgesprochen wesentlich mit. Wie - so läßt sich die Frage an einem weiteren Exempel stellen - wurde der Raketeningenieur Wernher von Braun, der vor 1945 an der Entwicklung der V1 und V2 für das nationalsozialistische Regime maßgeblich mitgewirkt hatte, nach dem Zweiten Weltkrieg in das deutsche Selbstbild eingepaßt, als Bundespräsident Heuss 1958 auf seinem Besuch in den USA mit dem nun dort für die amerikanische Weltraumforschung arbeitenden Braun zusammentraf ${ }^{29}$ ? Mit Blick auf die Mechanismen auswärtiger Repräsentation sind allgemein schließlich die möglichen Kontinuitäten deutscher Traditionen im Ausstellungs- und Messewesen zu beachten ${ }^{30}$.

Zeitlich liegt es nahe, sich auf die Periode vom Kalten Krieg bis zur Entspannungspolitik und der Aufnahme beider deutscher Staaten 1973 in die Vereinten Nationen zu konzentrieren, oder in wirtschaftlich-gesellschaftlichen Kategorien ausgedrückt: auf die Epoche von Wiederaufbau, Wirtschaftswunder und Wohl-

27 PA/AA, B2, 80, f. 312 u.ö.; siehe ferner BA [Bundesarchiv, Koblenz] B 136/7765 [Bundeskanzleramt, Aktenplanuntergruppe: Messen u. Ausstellungen im Ausland] Bd. 1.

28 Siehe zum Beispiel PA/AA, IIIA1, Bd. 207: BDI an Herren Mitglieder des Arbeitskreises „Runder Tisch“, Köln, 5. April 1965 (Betr.: Sitzung des BDI-Messearbeitskreises „Runder Tisch“ am 10.3.65).

29 Siehe Der Spiegel, 12/24 (11. Juni 1958) 16, sowie ebd. 12/7 (12. Febr. 1958) 36-42 und Titel; und das zugespitzte Interpretationsangebot als Old Shatterhand der Nachkriegszeit bei Jochim Radkau, "Wirtschaftswunder" ohne technologische Innovation? Technische Modernität in den 50er Jahren, in: Axel Schildt, Arnold Sywottek (Hrsg.), Modernisierung im Wiederaufbau. Die westdeutsche Gesellschaft der 50er Jahre (Bonn 1993, $\left.{ }^{2} 1998\right)$ 129-154, hier 146.

30 Siehe allgemein Ekkehard Mai, Expositionen. Geschichte und Kritik des Ausstellungswesens (München 1986), und besonders Hans-Ulrich Thamer, Geschichte und Propaganda. Kulturhistorische Ausstellungen in der NS-Zeit, in: GG 24 (1998) 349-381. 
standsgesellschaft bis zum Umbruch in der ersten Ölkrise von 1973. Unter Periodisierungsgesichtspunkten verspricht der (west)deutsche Fall besonders aufschlußreich zu sein. Folgt man der kürzlich von Charles S. Maier vorgeschlagenen strukturgeschichtlichen Einteilung, handelte es sich bei dem Zeitraum zwischen 1945 und den siebziger Jahren um die letzte Phase, in der ein begrenzt gedachtes (nationalstaatliches) Territorium allgemein als notwendige Voraussetzung für politische Macht, materielle Ressourcenausschöpfung und gesellschaftliche Identität angesehen wurde. Politisches Streben richtete sich demnach darauf, den Raum, der durch politische Entscheidungen zu kontrollieren war, mit dem Identitätsraum, auf den sich gesellschaftliche Loyalitäten bezogen, in Übereinstimmung zu bringen. Ab den späten sechziger Jahren verlor Maier zufolge das seit den $1860 \mathrm{er}$ Jahren beherrschende Konzept der Territorialität seine handlungsleitende Überzeugungskraft und Gültigkeit. Welche Rolle spielten diese Faktoren in unserem Zusammenhang: Stellte Deutschland aufgrund seiner Teilung einen Sonderfall dar, weil der Anspruch auf Wiedervereinigung die Fiktion von der möglichen Kongruenz des Identitätsraums mit dem Kontroll- und Entscheidungsraum perpetuierte? Oder nahm der westliche Teilstaat aufgrund seiner forcierten Integration in die westliche Welt frühzeitig Abschied von dem anderswo noch ausgeprägten Leitbild und bildete insofern lediglich eine der ersten Ausprägungen eines Typs postterritorialer Gesellschaftsordnung? Die Untersuchung auswärtiger Repräsentationen als reflexive Selbstwahrnehmung verspricht zur Beantwortung dieser Fragen einen wichtigen Beitrag zu leisten, gaben sie doch den vielfältigen Beziehungen über territoriale Grenzen hinweg sowie der Neubestimmung von Identitäten Ausdruck und formten sie zugleich.

Welcher Ansatz eignet sich, um die auswärtigen Repräsentationen und ibre Wirkungen auf das deutsche Selbstverständnis zu untersuchen? Nach den nationalsozialistischen Eroberungskriegen, dem Holocaust und der bedingungslosen Kapitulation bildete die Wiedereingliederung in die Welt eines der Hauptprobleme der Nachkriegszeit. Die Integration mußte auf allen Feldern von der Wirtschaft über Politik und Gesellschaft bis hin zur Kultur neu angegangen werden, und sie erfolgte unter den besonderen Bedingungen einer geteilten Welt. Die Aufgabe erforderte auf westdeutscher Seite eine Auseinandersetzung sowohl mit den internationalen Gegebenheiten wie auch mit den eigenen Voraussetzungen. Es handelte sich mithin um die exemplarische Situation eines Anpassungsvorgangs. Das Spektrum von Einstellungen reichte dabei von der Überzeugung über die Einsicht bis zum Gefühl, sich unter Zwang anpassen zu müssen ${ }^{31}$. Wie sollte man sich im

$31 \mathrm{Vgl}$. exemplarisch an drei machtpolitisch schwachen Staaten vor dem Ersten Weltkrieg Madeleine Herren, Hintertüren zur Macht. Internationalismus und modernisierungsorientierte Außenpolitik in Belgien, der Schweiz und den USA 1865-1914 (Studien zur Internationalen Geschichte 9, München 2000); vgl. auch Mary L. Dudziak, Cold War Civil Rights. Race and the image of American democracy (Princeton, NJ 2000), die zeigt, wie internationale Meinungsbildung und Kalter Krieg amerikanische Politiker und Beamte zwischen 1949 und dem Vietnamkrieg mit dazu bewogen, die Rassen- und Sozialpolitik im eigenen Land zu modifizieren. 
Umgang mit anderen verhalten, um seine Interessen wahren zu können, um akzeptiert zu werden? Wie ließen sich diese Interessen in einem demokratischen System überhaupt definieren? Sollte man fremde Ideen und Institutionen übernehmen? Welche eigenen Vorstellungen und Fähigkeiten erwiesen sich als nützlich, welche mußten besser verändert oder abgelegt werden? Die internationale Konfrontation im Kalten Krieg sowie die Dynamik weltweiter Entwicklungen führten in der zweiten Hälfte des 20. Jahrhunderts zu epochenspezifischen Antworten auf den allgemeinen Anpassungsdruck, in dessen Zusammenhang die Bundesrepublik keinen Einzelfall bildete, auch wenn Deutschland als Ganzes darüber hinaus durch seine staatliche Teilung innerhalb Europas zu einem besonderen Fall wurde.

Neuere geschichts- und sozialwissenschaftliche Untersuchungen zu transnationalen Bewegungen und Aktivitäten bieten methodische Anregungen für eine historische Analyse von internationalen Anpassungsprozessen. Ausgangspunkt ist die enger werdende Vernetzung der europäischen und später weltweiten Gesellschaften insbesondere seit dem Zeitalter der Aufklärung ${ }^{32}$. Zum einen haben ideengeschichtliche Forschungen hier kürzlich mit dem Konzept des interkulturellen Transfers eine für die Forschungspraxis fruchtbare Kategorie entwickelt, die den herkömmlichen, ,groben' Begriff des ,Einflusses' entbehrlich macht ${ }^{33}$. Die konkrete Vermittlung rückt ins Zentrum des Interesses, indem die Art und Weise, wie Wissen über ein Land in einen anderen Handlungs- und Deutungszusammenhang gelangt, analy tisch in einzelne Schritte zerlegt und so einer genauen Untersuchung zugänglich wird. Zum anderen haben sozialwissenschaftliche Untersuchungen transnationaler Phänomene sowie historische Arbeiten zur Internationalisierung seit der Mitte des 19. Jahrhunderts die strukturellen Bedingungen grenzüberschreitenden Handelns offengelegt ${ }^{34}$. Anstatt die ,große Politik' der europäischen

32 Die Kontakte erhielten im 19. Jahrhundert eine spezifisch nationalstaatliche und curopazentrierte Prägung, die erst im 20. Jahrhundert allmählich ihre Wirksamkeit einbüßte, so daß bis heute ein neues weltweites System entstanden ist; siehe den Überblick von Adam Watson, The evolution of international society. A comparative historical analysis (London 1992) Kap. 18-25; grundlegend Hedley Bull, Adam Watson (Hrsg.), The expansion of international society (Oxford 1984).

33 Grundlegend waren Arbeiten zum deutsch-französischen Kulturkontakt, die den Austausch von Literatur und Ideen sozialgeschichtlich erfaßten. Die Übertragungsprozesse sind prinzipiell auch für andere Elemente nationaler Kulturen beobachtbar (etwa für den Transfer von Technologie, aber auch von Sozialtechniken wie Sozialversicherungen) und die beteiligten Akteure stammen aus allen europäischen und zunehmend auch außereuropäischen Gesellschaften; siehe die Forschungsdiskussion bei Johannes Paulmann, Internationaler Vergleich und interkultureller Transfer. Zwei Forschungsansätze zur europäischen Geschichte des 18. bis 20. Jahrhunderts, in: HZ 267 (1998) 649-685 und Jürgen Osterhammel, Internationale Geschichte, Globalisierung und die Pluralität der Kulturen, in: Wilfried Loth, Jürgen Osterhammel (Hrsg.), Internationale Geschichte. Themen - Ergebnisse - Aussichten (München 2000) 387-408. Als neuere empirische Studie im europäisch-nordamerikanischen Raum, die lebhaft rezipiert worden ist, sei genannt Daniel T. Rogers, Atlantic crossings. Social Politics in a progressive age (Cambridge, MA 1998); zur Diskussion des Werks siehe das Review Symposium in H-DEMOG (Pfad: H-NET Discussion Logs / H-DEMOG / Symposium: Rodgers' Atlantic Crossings).

34 Siehe Thomas Risse-Kappen (Hrsg.), Bringing transnational relations back in. Non-state 
Kabinette isoliert zu betrachten und Nationalismus und Internationalismus als unvereinbare Gegensätze zu verstehen, wird das Zusammenwirken nationaler Bedingungen, internationalen Handelns und transnationaler Vorgänge systematisch erfaßt.

Diese methodischen Ansätze für die historische Analyse transnationaler und interkultureller Austauschbeziehungen berühren sich mit theoretischen Überlegungen zur „Modernisierung moderner Gesellschaften“. Dieser Vorgang beschäftigt nicht nur die Soziologie, sondern ist auch in der neueren historischen Forschung zur deutschen Geschichte seit der Wende zum 20. Jahrhundert immer wieder thematisiert worden ${ }^{35}$. Das hier skizzierte Forschungsprogramm zu den auswärtigen Repräsentationen und ihrer Wirkung auf das deutsche Selbstverständnis kann dabei an zwei grundlegende Vorgänge anknüpfen, die im sozialwissenschaftlichen Verständnis neben anderen die fortschreitende Moderne ausmachen: und zwar an die immer engere Verflechtung der Gesellschaften weltweit und an die Zunahme von Selbstreflexivität ${ }^{36}$. Es gilt, diese Vorgänge mit geschichtswissenschaftlichen Fragestellungen zu verbinden, sie zu historisieren, indem sie für ein bestimmtes Land in einem konkreten Kontext erforscht werden. Die reflexive Selbstwahrnehmung in auswärtigen Repräsentationen kann also auch als Teil einer

actors, domestic structures, and international institutions (Cambridge 1995) sowie aus historischer Sicht Geyer, Paulmann (Hrsg.), Mechanics of internationalism (wie Anm. 13).

${ }^{35}$ Siehe Wolfgang Zapf (Hrsg.), Die Modernisierung moderner Gesellschaften. Verhandlungen des deutschen Soziologentages in Frankfurt am Main 1990 (Frankfurt a.M. 1991) und das Schwerpunktheft „Modernisierung und Modernisierungstheorie“ von Leviathan 24/1 (1996) 8-108. In der historischen Forschung tauchte die Frage nach der Modernität Deutschlands in verschiedener Gestalt auf: in der Sonderwegsdebatte, im Streit um die Modernisierung durch den Nationalsozialismus und schließlich im Zusammenhang der Diskussion um Kontinuität und Neubeginn nach dem Zweiten Weltkrieg. Für die Entwicklung der Bundesrepublik bis in die siebziger Jahre liegen sehr unterschiedliche Bewertungen vor, die vom Vorwurf der Restauration über die Kennzeichnung als konservative Modernisierung bis hin zur emphatischen Charakterisierung als aufregende Modernisierung reichen; siehe Hans-Peter Schwarz, Modernisierung oder Restauration? Einige Vorfragen zur künftigen Sozialgeschichtsschreibung über die Arra Adenauer, in: Kurt Düzell, Wolfgang Köllmann (Hrsg.), Rheinland-Westfalen im Industriezeitalter 3: Vom Ende der Weimarer Republik bis zum Land NordrheinWestfalen (Wuppertal 1984) 278-293; Hans Günter Hockerts, Das Ende der Ära Adenauer. Zur Periodisierung der Bundesrepublikgeschichte, in: Winfried Becker, Werner Chroback (Hrsg.), Staat, Kultur, Politik. Beiträge zur Geschichte Bayerns und des Katholizismus. (Fs. Dieter Albrecht, Kallmünz 1992) 461-475; Arnold Sywottek, Wege in die 50er Jahre, in: Schildt, Sywottek (Hrsg.), Modernisierung im Wiederaufbau (wie Anm. 29) 13-39; und Michael Prinz, Demokratische Stabilisierung, Problemlagen von Modernisierung im Selbstbezug und historische Kontinuität - Leitbegriffe einer Zeitsozialgeschichte, in: Westfälische Forschungen 43 (1993) 655-675.

36 Siehe die durchaus unterschiedlich akzentuierten Positionen in Ulrich Beck, Anthony Giddens, Scott Lash, Reflexive modernization. Politics, tradition and aesthetics in the modern social order (Cambridge 1994) bes. 1-4, 95-107, 110-119 und 175-178; zu Giddens siehe auch Thomas Mergel, Geht es weiterhin voran? Die Modernisierungstheorie auf dem Weg zu einer Theorie der Moderne, in: ders., Thomas Welskopp (Hrsg.), Geschichte zwischen Kultur und Gesellschaft. Beiträge zur Theoriedebatte (München 1997) 203-232. 
gesellschaftlichen Modernisierung nach den Erfahrungen des Zweiten Weltkriegs verstanden werden ${ }^{37}$.

Angeregt von diesen neueren methodischen Ansätzen und theoretischen Überlegungen ergeben sich folgende Leitfragen für die Untersuchung auswärtiger Repräsentationen und reflexiver Selbstwahrnehmung in Deutschland:

(1) Wer waren die Träger der auswärtigen Repräsentationen und was bewegte sie? An der westdeutschen Selbstdarstellung im Ausland beteiligten sich verschiedene Institutionen, Gruppen und Persönlichkeiten. Das Spektrum reichte von offiziellen Regierungsstellen über halbamtliche Einrichtungen bis hin zu privaten Initiativen. Motive und Interessen wichen durchaus voneinander ab. Die internationalen Aktivitäten von regierungsamtlichen und nichtregierungsamtlichen Organisationen, Wirtschaftsunternehmen oder Expertenvereinigungen mußten nicht alle denselben, national- bzw. teilstaatlichen Zielen dienen. Ebensowenig ist aber von einer scharfen Trennung zwischen amtlichen und gesellschaftlichen Trägern auszugehen, sondern vielmehr danach zu fragen, wie sich die Zusammenarbeit institutionell entwickelte, welche Konflikte auftraten und wie sie gegebenenfalls gelöst wurden. Da die Diskussionen innerhalb der Bundesrepublik über die Präsentation Deutschlands in der Welt im Mittelpunkt stehen, erfaßt ihre Untersuchung auch die Haltung gesellschaftlicher Gruppen, die im Rahmen internationaler Beziehungen gewöhnlich wenig Beachtung finden, wie etwa diejenige der Architekten und Designer von Ausstellungspavillons. Lassen sich schließlich $A \mathrm{k}-$ teure in Deutschland identifizieren, die jenseits institutioneller, generationeller, sozialer oder politischer Schranken internationalistisch aktiv waren?

(2) Wie verlief der reflexive Transferprozeß der Selbstdarstellung im Ausland? Die westdeutsche Diskussion um die auswärtige Repräsentation ist als ein "Wechselspiel der Eigenwahrnehmung “ oder, präziser ausgedrückt, als reflexiver Transfer zu verstehen ${ }^{38}$. Zugrunde lagen zwei verschiedene Transferakte, die sich analytisch voneinander trennen lassen, auch wenn sie in der historischen Situation eng miteinander verwoben waren: zum einen die Darstellung des eigenen Landes im Ausland, zum anderen die Rückvermittlung dieser Präsentation in die Bundesrepublik. Am Anfang stand die bewußte Auswahl von materiellen und immateriellen Dingen, die in einem fremden Land oder auf einer internationalen Messe bzw. Ausstellung vorgestellt werden sollten. Dem folgten Entscheidungen über die Art der Darstellung, d.h. eine Umsetzung in Wort und Bild, die gegebenenfalls durch Übersetzung den besonderen Umständen am fremden Ort angepaßt wurde. Dies

37 Vgl. Michael Pugh (Hrsg.), Regeneration of war-torn societies (Global issues series, New York 2000).

38 Siehe für die Zerlegung von Transferakten in einzelne Schritte Rudolf Mubs, Johannes Paulmann, Willibald Steinmetz, Brücken über den Kanal? Interkultureller Transfer Zwischen Deutschland und Großbritannien im 19. Jahrhundert, in: dies. (Hrsg.), Aneignung und Abwehr. Interkultureller Transfer zwischen Deutschland und Großbritannien im 19. Jahrhundert (Bodenheim: Philo 1998) 7-20, sowie Johannes Paulmann, Interkultureller Transfer zwischen Deutschland und Großbritannien: Einführung in ein Forschungskonzept, ebd. 2143. 
war der erste Akt des Transfers. Der zweite Teil begann mit den Kommentaren der anderen, die auf unterschiedlichen Wegen selektiv nach Westdeutschland vermittelt wurden. Im Mittelpunkt des Interesses steht allerdings nicht das Bild, das die fremden Beobachter von der Bundesrepublik besaßen, sondern die internen Reaktionen auf das Selbstbild, das dem Ausland vorgeführt worden war. Einzelne Teile oder die gesamte Präsentation wurden Gegenstand politischer Debatten in der Bundesrepublik. Sie fügten sich in aktuelle Argumentations- und Handlungszusammenhänge ein und konnten unter Umständen künftige Selbstdarstellungen verändern. Die Diskussion um die auswärtigen Repräsentationen bildete so einen wesentlichen Teil der Selbstvergewisserung einer sich formierenden bundesrepublikanischen Gesellschaft.

(3) Wie entwickelte sich das Selbstverständnis in der Bundesrepublik im Rahmen der Systemkonkurrenz? Die Reflexion über die Integration der Bundesrepublik in die Welt kann nicht nach dem Schema, hier Deutschland, dort die Welt' untersucht werden. Während andere europäische Länder die Entwicklung einer internationalen Gesellschaft nach dem Zweiten Weltkrieg als Nationalstaaten mitgestalteten, beteiligte Deutschland sich aufgrund seiner staatlichen Spaltung unter anderen Voraussetzungen. Die Selbstdarstellung der DDR im Ausland bildete als Konkurrenzunternehmen aus der Sicht der Bundesrepublik eine unverzichtbare Folie: zeitgenössisch für die Westdeutschen, deren Auftreten sie entscheidend beeinflußte, und aus historischer Sicht als Vergleichsobjekt. Der Bezug auf eine gemeinsame deutsche Vergangenheit und insbesondere die erforderliche Abkehr von der unmittelbar vorausgegangenen Epoche des Nationalsozialismus waren nur ein Aspekt im Rahmen der Veränderung des Selbstverständnisses ${ }^{39}$. Die Abwehr dessen, wofür der andere deutsche Staat stand, prägte die auswärtigen Rcpräsentationen mindestens ebenso. Abkehr und Abwehr überlagerten sich gegenseitig und verstärkten einander im Einzelfall oder schwächten sich ab. Die Brüche und Teilungen der deutschen Nachkriegsgeschichte steigerten allgemein, so ist anzunehmen, im Rahmen der Eigendarstellung die Selbstreflexivität. Zudem konnten sie bewirken, daß Modernität sich - u.a. im Gewand von Internationalität - zu einer besonders attraktiven Zielvorstellung entwickelte, die den Mangel an historischer und nationaler Kontinuität kompensieren half.

(4) Wie veränderte sich bundesrepublikanisches Selbstverständnis im Zeichen von Internationalisierung, europäischer Integration und Globalisierung? Der gegenwärtige Gebrauch des Schlagworts, Globalisierung' suggeriert häufig, daß eine Welt (bereits) existiere und lokale Kulturen durch die zugrundeliegenden ökonomischen Prozesse vereinheitlicht würden. Aus historischer Sicht bleibt aber die darin enthaltene Entwicklungsperspektive genauer zu untersuchen ${ }^{40}$. Richteten sich die auswärtigen Repräsentationen der Bundesrepublik und das Bemühen um

39) Vgl. Mary Fulbrook, German national identity after the Holocaust (Oxford 1999).

to Vgl. die anthropologische Untersuchung von Lokalisierungsvorgängen in fünf verschicdenen Orten Asiens in James L. Watson (Hrsg.), Golden Arches East. McDonald's in East Asia (Stanford, CA 1997). 
Integration zwischen Kaltem Krieg und Entspannungspolitik überhaupt auf ,die Welt'? Zu unterscheiden ist zwischen verschiedenen auswärtigen Bezügen, in denen die Selbstdarstellung stattfand: der internationalen Ebene im zwischenstaatlichen, gegebenenfalls allianzpolitischen Sinne, den transnationalen Zusammenhängen und der europäischen Integrationsbewegung. Wie im deutsch-deutschen Verhältnis sind auch hier die möglichen Folgen von Überschneidungen zu beden$k_{e n}{ }^{41}$. Weiter ist zu fragen, wer ab wann, wenn überhaupt, erwartete, daß einmal eine internationale Gesellschaft entstehen würde. Entwickelten sich die diesbezüglichen Vorstellungen in Politik, Wirtschaft, Gesellschaft und Kultur gleichzeitig oder gab es hier Bereiche und Gruppierungen, in denen dies früher geschah als in anderen ${ }^{42}$ ? Schließlich gilt es zu erörtern, wie sich auswärtige Repräsentationen angesichts des Wandels in der Welt veränderten, denn die Bundesrepublik und ihre Gesellschaft integrierte sich nicht in eine ruhende, sondern in eine dynamische internationale Umgebung. Hingewiesen sei etwa auf die ,Dritte Welt', die als Ort westdeutscher und deutsch-deutsch konkurrierender Selbstdarstellung erst im Laufe der Nachkriegsjahrzehnte Bedeutung gewann. Im Rahmen der Entwicklungspolitik konnten deutsche Vertreter dort zugleich ihre eigenen Vorstellungen von Modernität bestätigt finden und sich so im Vergleich über ihre eigene Position selbst vergewissern.

Insgesamt helfen die soeben erläuterten Leitfragen zu klären, wie bewußte internationale Aktivität und die damit verknüpfte reflexive Eigenwahrnehmung kulturelle, soziale und politische Verhaltens- und Wahrnehmungsmuster in Westdeutschland veränderten. Was trugen auswärtige Repräsentationen zu einer bundesrepublikanischen Mentalität bei? Sind „Westernisierung“ oder Amerikanisierung angemessene Beschreibungen für dic auf diesem Weg eingeübten Verhaltensweisen ${ }^{43}$ ? Gab es Kontinuitätslinien zur deutschen Vergangenheit und welche Bezichungen zum konkurrierenden System wirkten ein? Läßt sich im besonderen Verhältnis der Bundesrepublik zur Welt eine spezifische Auffassung von Modernität erkennen?

$41 \mathrm{Vgl}$. Hartmut Kaelble, Supranationalität in Europa seit dem Zweiten Weltkrieg. Einleitende Bemerkungen, in: Heinrich August Winkler, Hartmut Kaelble (Hrsg.), Nationalismus - Nationalitäten - Supranationalität (Stuttgart 1993) 189-206; und Alexander Schmidt-Gernig, Gibt es eine „europäische Identität“? Konzeptionelle Überlegungen zum Zusammenhang transnationaler Erfahrungsräume, kollektiver Identitäten und öffentlicher Diskurse in Westeuropa seit dem Zwciten Weltkrieg, in: Hartmut Kaelble, Jürgen Scbriewer (Hrsg.), Diskurse und Entwicklungspfade. Der Gesellschaftsvergleich in den Geschichts- und Sozialwissenschaften (Frankfurt a.M. 1999) 163-216.

42 Als geradezu gegenläufig und, besonders nach 1945, eng verknüpft mit der Abwehr transnationaler Entwicklungen erweist sich die anhaltende Repräsentation des Deutschen in Vorstellungen von "Heimat"; siche Celia Applegate, A nation of provincials. The German idea of Heimat (Berkeley, CA 1990) bes. 228-246, und Alon Confino, Edgar Reitz's „Heimat“ and the German nationhood. Film, memory, and understandigs of the past, in: German History $16(1998)$ 185-208.

$43 \mathrm{Vgl}$. Anselm Doering-Manteuffel, Wie westlich sind dic Deutschen? Amerikanisierung und Westernisierung im 20. Jahrhundert (Göttingen 1999), und Febrenbach, Poiger (Hrsg.), Transactions, transgression, transformations (wie Anm. 9) xiii-xl. 
Abschließend sei der prinzipielle Erkenntnisgewinn des hier skizzierten Forschungsprogramms zu den auswärtigen Repräsentationen der Bundesrepublik hervorgehoben. Es stellt die deutsche Geschichte nachdrücklich in einen transnationalen Zusammenhang, in dem diese prinzipiell nicht nur als Einzelfall, sondern als Beispiel und möglicherweise sogar als ein Typ zu bestimmen sein wird. Auf den Ergebnissen der Forschungen zur Außenpolitik aufbauend und sie wesentlich ergänzend, werden wirtschaftliche, soziale und kulturelle Entwicklungen des westdeutschen Teilstaats aufgegriffen und im Wechselspiel der Eigenwahrnehmung untersucht. Die Konstruktion eines Selbstbildes der Bundesrepublik steht dabei im Bezug sowohl zum Ausland als auch zur DDR. Die Anpassung an fremde Verhältnisse und zugleich das Bewahren oder Verändern hergebrachter Verhaltensweisen gerät ins Blickfeld. Abkehr, Abwehr und Modernität werden in ihrer gegenseitigen Verflechtung analysiert.

In den auswärtigen Repräsentationen ist die Geschichte der internationalen Beziehungen eng mit der Kultur- und Mentalitätsgeschichte verbunden, so daß zumeist getrennt betrachtete Erscheinungen der inneren und äußeren Zeitgeschichte miteinander verknüpft werden können. Zugleich ermöglicht die Auswahl der konkreten Untersuchungsgegenstände ökonomische Handlungsfelder, wie Industriemessen, mit kulturellen Bereichen, wie der Arbeit von Goethe-Instituten, unter einer gemeinsamen Fragestellung zu behandeln. Schließlich sind von dem Forschungsprogramm historische Erkenntnisse über zentrale Vorgänge in den knapp drei Jahrzehnten nach dem Zweiten Weltkrieg zu erwarten, deren Auswirkungen bis in gegenwärtige Debatten um deutsches Selbstverständnis sowie um Stellung und Auftreten Deutschlands in der Welt reichen. Ob die eingangs geschilderte Haltung der Zurückhaltung als eines der charakteristischen Momente, das in der Nachkriegszeit ausgeformt wurde, weiterhin kennzeichnend bleiben oder aber von anderen Verhaltensnormen verdrängt werden wird, bleibt hingegen abzuwarten. 